\title{
Wave normal angles of magnetospheric chorus emissions observed on the Polar spacecraft
}

\author{
N. Haque, ${ }^{1}$ M. Spasojevic, ${ }^{1}$ O. Santolík, ${ }^{2,3,4}$ and U. S. Inan ${ }^{1,5}$ \\ Received 31 July 2009; revised 26 October 2009; accepted 3 November 2009; published 1 April 2010.
}

[1] Using data from the High Frequency Waveform Receiver on board the Polar spacecraft, 1,765 and 993 wave normal angles have been analyzed for 13 orbits containing upper band magnetospheric chorus emissions and 15 orbits containing lower band emissions, respectively. The purpose of this study is to characterize the distribution of the polar wave normal angle, $\theta$, for chorus emissions as a function of magnetic latitude, $\lambda$. Understanding wave normal angles is an important step in evaluating resonant waveparticle interactions. For upper band chorus, wave normal angles tend to remain at or rise toward the resonance cone angle for low latitudes and midlatitudes but move away from the resonance cone angle at higher latitudes. For lower band chorus, wave normal angles with values $\theta<20^{\circ}$ have the highest probability of occurrence in the latitude range of $10^{\circ}-50^{\circ}$. Just off the equator, $10^{\circ} \leq \lambda<25^{\circ}$, there exists a secondary occurrence peak in the range of $50^{\circ} \leq \theta<70^{\circ}$. The probability of observing these higher wave normal angles decreases with increasing latitude. The time-averaged Poynting flux, $S$, is much larger for lower band chorus waves, which have a mean value of $8.5 \times 10^{-8} \mathrm{~W} / \mathrm{m}^{2}$, than for upper band chorus waves, which have a mean value of $1.4 \times 10^{-9} \mathrm{~W} / \mathrm{m}^{2} . S$ is fairly evenly distributed about its median value, $3.1 \times 10^{-10} \mathrm{~W} / \mathrm{m}^{2}$, for all wave normal angles for upper band chorus but deceases as $\theta$ increases for lower band chorus.

Citation: Haque, N., M. Spasojevic, O. Santolík, and U. S. Inan (2010), Wave normal angles of magnetospheric chorus emissions observed on the Polar spacecraft, J. Geophys. Res., 115, A00F07, doi:10.1029/2009JA014717.

\section{Introduction}

[2] Whistler mode chorus waves are one of the most common and most intense natural plasma waves occurring in the Earth's outer magnetosphere [Storey, 1953; Allcock, 1957; Helliwell, 1965, 1969]. Usually observed in the region outside of the plasmapause, chorus waves are discrete emissions often containing rising and falling tones, as well as short impulsive bursts [Burtis and Helliwell, 1969; Dunckel and Helliwell, 1969; Burton and Holzer, 1974; Sazhin and Hayakawa, 1992; LeDocq et al., 1998; Lauben et al., 2002]. Chorus occurs over a broad frequency range, from hundreds of $\mathrm{Hz}$ up to about $10 \mathrm{kHz}$ [Gurnett and O'Brien, 1964]. Within this range, chorus waves typically occur in two distinct frequency bands, a lower band with frequencies $0.1 f_{c e-e q} \leq f<0.5 f_{c e-e q}$ and an upper band with frequencies $0.5 f_{c e-e q} \leq f \leq 0.65 f_{c e-e q}$, where $f_{c e-e q}$ denotes the equatorial gyrofrequency. There is typically a gap between

\footnotetext{
${ }^{1}$ Space, Telecommunications, and Radioscience Laboratory, Stanford University, Stanford, California, USA.

${ }^{2}$ Department of Physics and Astronomy, University of Iowa, Iowa City, Iowa, USA.

${ }^{3}$ Permanently at Department of Space Physics, Institute of Atmospheric Physics, Prague, Czech Republic.

${ }^{4}$ Permanently at Faculty of Mathematics and Physics, Charles University, Prague, Czech Republic.

${ }^{5}$ Koç University, Istanbul, Turkey.

Copyright 2010 by the American Geophysical Union. 0148-0227/10/2009JA014717
}

these two bands at $0.5 f_{\text {ce-eq }}$, where the wave power is at a minimum [Burtis and Helliwell, 1969, 1976; Tsurutani and Smith, 1974].

[3] Chorus is believed to play a major role in the evolution of energetic particle distributions in the magnetosphere. Cyclotron resonant wave-particle interactions involving chorus can result in the precipitative loss of electrons over a wide range of energies. Chorus is believed to be a driver of low-energy $(<1 \mathrm{keV})$ diffuse auroral precipitation [Inan et al., 1992; Villalon and Burke, 1995; Ni et al., 2008], medium energy (10s to $100 \mathrm{keV}$ ) microburst precipitation [Rosenberg et al., 1971, 1981, 1990], and relativistic $(>1 \mathrm{MeV})$ microburst precipitation [Lorentzen et al., 2001; Thorne et al., 2005]. Interactions with chorus can also result in energy diffusion and thus contribute to the acceleration of electrons to $\mathrm{MeV}$ energies [Horne and Thorne, 1998; Horne et al., 2005a, 2005b; Shprits et al., 2006; Summers et al., 2007a, 2007b].

[4] Pitch angle scattering and energy diffusion can occur when the resonance condition, $\omega-k_{\|} v_{\|}=\frac{m \omega_{c e}}{\gamma}, m=0, \pm 1$, $\pm 2, \pm 3, \ldots$, is satisfied. In this expression, $\omega$ is the frequency of the wave, $k_{\|}$and $v_{\|}$are the components of the wave vector and particle velocity parallel to the ambient magnetic field, respectively, $m$ is the resonance harmonic number, $\omega_{c e}$ is the local gyrofrequency, and $\gamma=\left(1-\frac{v^{2}}{c^{2}}\right)^{-\frac{1}{2}}$ is the relativistic correction factor, where $v$ is the particle velocity and $c$ is the speed of light [Kennel and Petschek, 1966]. The wave vector is typically specified with respect to the direction 


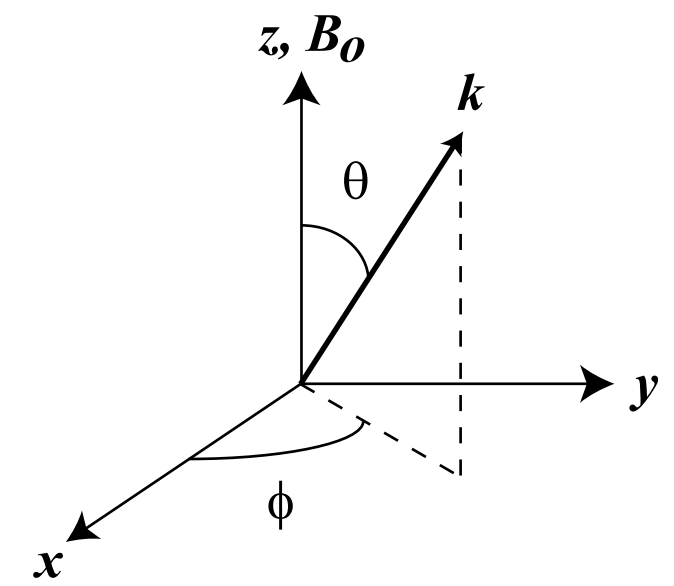

Figure 1. Coordinate system depicting polar wave normal angle, $\theta$, azimuthal wave normal angle, $\phi$, and wave vector, $k$. The direction of the local magnetic field, $B_{o}$, is assumed to be in the $z$ direction in this system.

of the local magnetic field, $B_{o}$, with the polar wave normal angle, $\theta$, defined as the angle between $B_{o}$ and the wave vector (from $0^{\circ}$ to $180^{\circ}$ ) and the azimuthal wave normal angle, $\phi$, measured about the direction of $B_{o}$ (from $-180^{\circ}$ to $180^{\circ}$ ). This coordinate system is illustrated in Figure 1, with the direction of $B_{o}$ assumed to be in the $z$ direction. The polar wave normal angle is needed to evaluate the resonance condition and thus is the focus of the current study. On the other hand, both $\theta$ and $\phi$ are needed to predict how the wave will propagate in the magnetosphere from its current location.

[5] While there have been several previous studies of chorus wave normal angles, many of them focused on the generation mechanism of chorus. Consequently, these studies were mostly limited to the equatorial plane, which has been shown to be the source region for chorus waves throughout the inner magnetosphere [LeDocq et al., 1998; Parrot et al., 2003; Santolik et al., 2005]. Past studies of wave normal angles for upper band chorus emissions near the magnetic equator, $\lambda<5^{\circ}$, show different results. (Note: Here and in the remainder of the paper, the symbol $\lambda$ refers to the absolute value of the magnetic latitude.) From studying one pass of the GEOS 2 satellite, Hayakawa et al. [1984] concluded that the wave normal angles are near the resonance cone. However, Hospodarsky et al. [2001] found that the emissions propagate primarily parallel to the magnetic field from the analysis of one pass of the Cassini spacecraft. Lauben et al. [2002] also showed that upper band chorus was emitted at $\theta \sim 0^{\circ}$ near the equator using properties of the wave measured by the Polar satellite and then doing multifrequency ray tracing back to the source region. Outside the equatorial plane, Muto et al. [1987] analyzed two passes of the GEOS 1 satellite and concluded that $\theta$ is close to $\theta_{\text {res }}$ for $\lambda \sim 17^{\circ}$, but that $\theta$ is $15^{\circ}-20^{\circ}$ less than $\theta_{\text {res }}$ for $\lambda \sim 26^{\circ}$.

[6] Studies of lower band chorus emissions in the equatorial plane, $\lambda<6^{\circ}$, had some similarities. From three passes of the OGO 5 satellite, Goldstein and Tsurutani [1984] found that the majority of wave normal angles for lower band chorus had values $\theta<20^{\circ}$, as did Hayakawa et al. [1984] for the lower band rising emissions with small values of $d f / d t$. However, Hayakawa et al. [1984] found $\theta=$ $30^{\circ}-45^{\circ}$ for lower band risers with increased values of $d f / d t$. Hospodarsky et al. [2001] concluded that lower band emissions have $\theta \simeq 0^{\circ}$ in this latitude region. However, Lauben et al. [2002] deduced that lower band chorus waves are emitted with wave normal angles close to the Gendrin angle, defined as the nonzero wave normal angle for which the wave propagates parallel to the magnetic field. Burton and Holzer [1974] studied 18 passes of the OGO 5 satellite containing lower band chorus over a much broader range of magnetic latitudes. They concluded that $\theta<30^{\circ}$ for $80 \%$ of the wave normal angles occurring in $\lambda<40^{\circ}$. However, in the higher-latitude region $\left(\lambda=40^{\circ}-50^{\circ}\right)$, the wave normals were more broadly distributed, extending to $85^{\circ}$.

[7] Because of the difficulty in determining chorus wave normal angles, as measurements are required of all three magnetic field components and at least two electric field components, the previous studies were not only confined to a small region of the magnetosphere, but also very limited in the number of wave normal angles analyzed. The goal of the present study is to establish a broader understanding of chorus wave normal angles, as determined from measurements of the three-component electric and magnetic field observed on the Polar spacecraft, not only in the source region but for wherever chorus waves propagate. To achieve this goal, the present study analyzes 1,765 wave normal angles for upper band chorus emissions and 993 wave normal angles for lower band chorus emissions over a broad range of magnetic latitudes, in both the equatorial and offequatorial regions, $L$ shells, and magnetic local times. The results of this study are important for studies of resonant interactions between chorus waves and electrons over a wide range of energies.

\section{Polar Spacecraft}

[8] The data used in the present study of magnetospheric chorus wave normal analysis were obtained by the Polar spacecraft. Launched on 24 February 1996, the Polar spacecraft has a highly elliptical, $86^{\circ}$ inclination orbit with a period of approximately $17.5 \mathrm{~h}$, perigee of $1.8 R_{\mathrm{E}}$, and apogee of $9 R_{\mathrm{E}}$. The Plasma Wave Instrument (PWI), on board the Polar spacecraft, provides high-resolution plasma wave data within the chorus band. The PWI detects magnetic fields through the use of a triaxial magnetic search coil antenna (70 $\mu \mathrm{V} / \mathrm{nT}-\mathrm{Hz}$ sensitivity) and detects electric fields using three orthogonal electric dipole antennas, two in the spacecraft spin plane (100 and $130 \mathrm{~m}, 6 \mathrm{~s}$ period), and one aligned along the spin axis $(14 \mathrm{~m})$. The signals from these antennas are processed by several receiver systems, one of which is the High Frequency Waveform Receiver (HFWR). Coincident sampling from $20 \mathrm{~Hz}$ to $25 \mathrm{kHz}$ of 3 orthogonal components of the electric and magnetic wave fields is provided by the HFWR. In the high-telemetry rate mode, $0.45 \mathrm{~s}$ waveform snapshots are recorded from each antenna at intervals spaced $9.2 \mathrm{~s}$ apart [Gurnett et al., 1995]. The present study looks at a subset of 59 orbits containing chorus, as determined by LeDocq et al. [1998] through the examination of all available high-telemetry HFWR data, consisting of 394 orbits from 28 March 1996 to 16 September 1997, after which the PWI instrument ceased to operate.

[9] For all of these orbits, the local gyrofrequency was obtained from measurements of the magnetic field from the 
Magnetic Field Experiment on board Polar [Russell et al., 1995]. For the orbits for which data was available, the PWI Sweep Frequency Receiver (SFR) was used to extract the electron density from the upper hybrid resonance line [Mosier et al., 1973]. The SFR, which covers frequencies in the range of $26 \mathrm{~Hz}$ to $808 \mathrm{kHz}$ at higher than one minute resolution, provides continuous data of a single component of the electric and magnetic fields [Gurnett et al., 1995].

\section{Computation of Chorus Wave Normal Angles Using Singular Value Decomposition}

[10] The chorus wave normal angles used in this study were obtained using a preprocessed data set. A sample plot of this data for an orbit containing lower band chorus from 1952 to 2003 UT and upper band chorus from 1957 to 2010 UT on 14 December 1996 is shown in Figure 2. All of the measured and calculated parameters of Figure 2 have a log spaced frequency resolution ranging from $9.2 \mathrm{~Hz}$ to $1.2 \mathrm{kHz}$ for 123 frequencies ranging from 0.2 to $25 \mathrm{kHz}$ and a time resolution of $0.45 \mathrm{~s}$. As previously stated, each antenna only records $0.45 \mathrm{~s}$ snapshots of the waveform every $9.2 \mathrm{~s}$. Therefore, the data in Figure 2 is not actually continuous but is the concatenation of each of the $0.45 \mathrm{~s}$ snapshots expanded over a $9.2 \mathrm{~s}$ interval.

[11] Figures 2 (first panel) and 2 (second panel) represent measured quantities of the sum of the three magnetic autopower spectra and the sum of the three electric autopower spectra. The singular value decomposition (SVD) technique described by Santolik et al. [2003] is used to further analyze the multicomponent wave measurements. SVD is used on the electromagnetic spectral matrix to determine the polar wave normal angle, $\theta$, the azimuthal wave normal angle, $\phi$ [Santolik et al., 2003, equation (22)], and the electromagnetic planarity, $F_{E}$, which determines the planarity of the electromagnetic wave [Santolik et al., 2003, equation (31)]. SVD is also used on the magnetic spectral matrix to obtain the degree of planar polarization, $P$ (see Santolik and Gurnett [2002] or equation (A6) in the appendix of Santolik et al. [2002]) and the ellipticity of polarization, $E_{\theta}$ (see appendix of Santolik et al. [2002]). Values of $P$ close to 1.0 mean that the magnetic field of the wave is coherent and that the ellipticity parameter is well defined.

[12] The present study required $P>0.9$ to ensure that $E_{\theta}$ is well defined. In order for the waves to meet the right hand circular polarization condition for whistler mode waves, it was required that $E_{\theta}>0.8 . F_{E}>0.8$ was also required to ensure that the electromagnetic wave was close to a single plane wave and that the wave normal angles calculated are valid for whistler mode waves.

\section{Classification of Wave Normal Angles}

[13] The next step in the analysis of the wave normal angles is to separate upper band and lower band chorus emissions. Past work has shown that the chorus wave frequency is more closely associated with the equatorial gyrofrequency than the local gyrofrequency, with upper band chorus located at frequencies just above $0.5 f_{c e-e q}$ and lower band chorus located in the range 0.1 to $0.5 f_{c e-e q}$ [e.g., Burtis and Helliwell, 1969]. In addition, when both emissions are observed at the same time, there tends to be a narrow but distinguishable gap between the two bands. Thus, in order to distinguish between upper and lower band chorus, we first estimate the equatorial gyrofrequency. The equatorial gyrofrequency was estimated by scaling the local gyrofrequency, calculated using the local magnetic field measured by the onboard magnetometer, using the current magnetic latitude and $L$ shell of the spacecraft and assuming a dipolar variation of the field strength along the field line. In all of the plots of Figure 2, the solid black lines represent 0.1 and 0.5 times the estimated equatorial gyrofrequency. However, since $f_{c e-e q}$ was estimated based on a dipolar scaling, there are likely to be errors when operating at high latitudes and $L$ shells. The maximum error in $f_{c e-e q}$ is estimated to be on the order of $\sim 15-20 \%$, and therefore, the estimated $f_{c e-e q}$ was used only as a starting guide to distinguish between the two bands. Visual inspection of the electric field for each individual orbit was used to separate the two bands along the line that best fit the typical gap seen between the two bands to ensure minimal overlap between the bands. The electric field was used to make the distinction because the strength of the electric field is typically on the order of at least two magnitudes larger than the strength of the magnetic field, making it easier to see the gap between the two bands visually. For instance, in Figure 2, though the upper solid black line representing $0.5 f_{c e-e q}$ is used as a starting guide to distinguish between the upper and lower band emissions, visual inspection of the electric field shows that the typical gap between the two bands actually occurs below this line. The line that best fit this typical gap was manually found for this and every other orbit used in this study. Using this method, it was determined that 24 orbits contained upper band chorus emissions, while 35 orbits contained lower band chorus emissions.

[14] Due to its highly elliptical orbit, the Polar spacecraft crosses the magnetic equator in a region just inside the plasmapause at $L$ shells ranging from 2.5 to 4.5 . For magnetic latitudes $\lambda<30^{\circ}$, the spacecraft typically samples $L$ shells up to $L=8$ [Gurnett et al., 1995]. Thus, in analyzing the wave normal angles calculated from Polar data, it is impossible to distinguish between latitudinal and $L$ shell effects. However, the equatorial plane has been shown to be the source region of the waves, and no current theories for wave generation are $L$ shell dependent. Thus, the propagation of the waves from the equatorial to the off-equatorial region is expected to have a much stronger effect on the wave normal angle distribution than variations with $L$ shell. Therefore, the variation of wave normal angle in this study will be characterized only in terms of magnetic latitude.

[15] When the strength of the total electric field, $E$, is weak for the chorus emissions, there exists an immense amount of scatter and variation in the wave normal angles as a function of magnetic latitude. In order to reduce some of the scatter attributed to noisy measurements, a frequencydependent threshold level was determined. Thus, the orbits

Figure 2. Sample plots of data for orbit containing lower band chorus from 1952 to 2003 UT and upper band chorus from 1957 to 2010 UT on 14 December 1996. The two solid lines in each plot represent 0.1 and 0.5 times the equatorial gyrofrequency. 


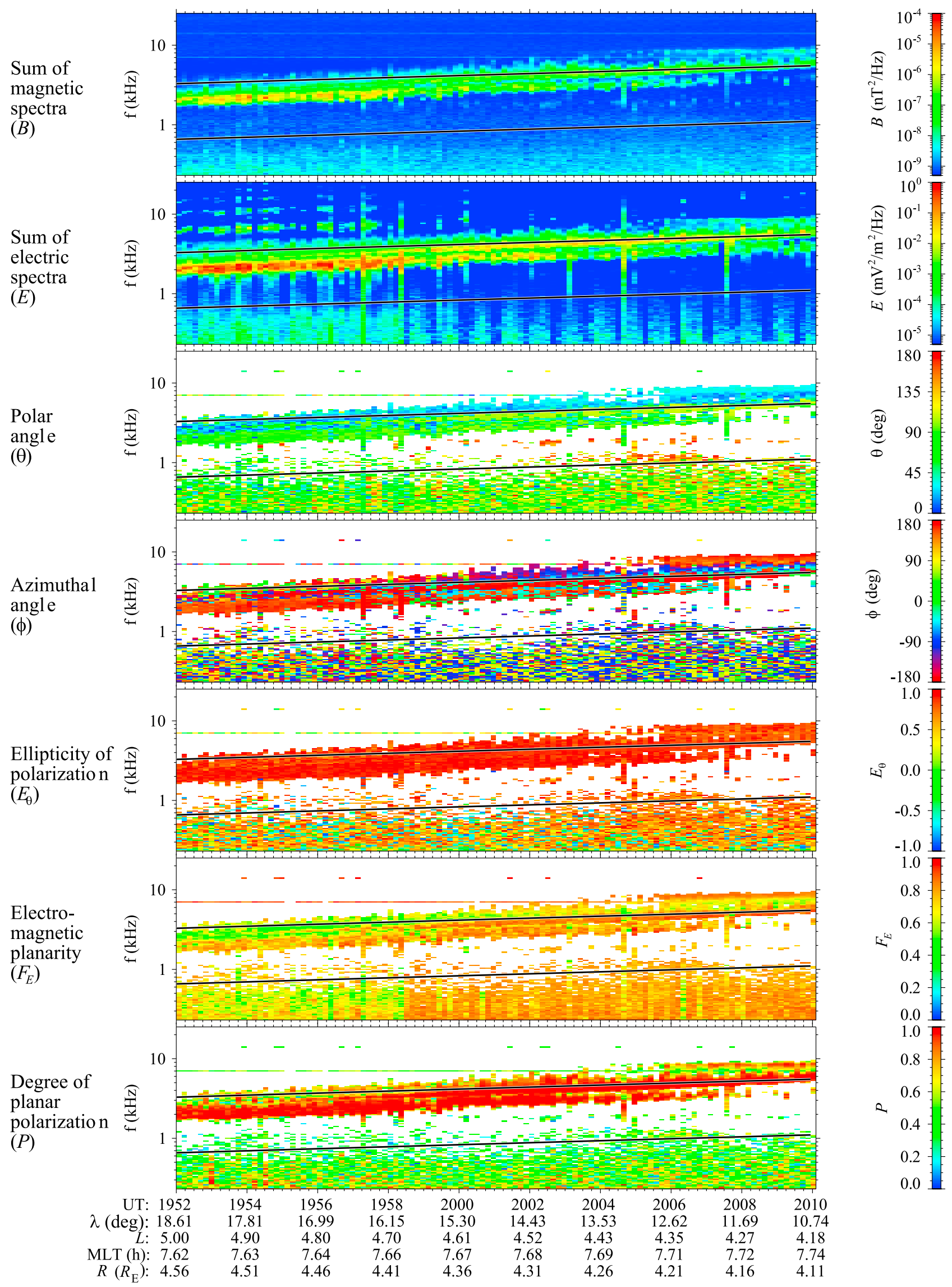

Figure 2 

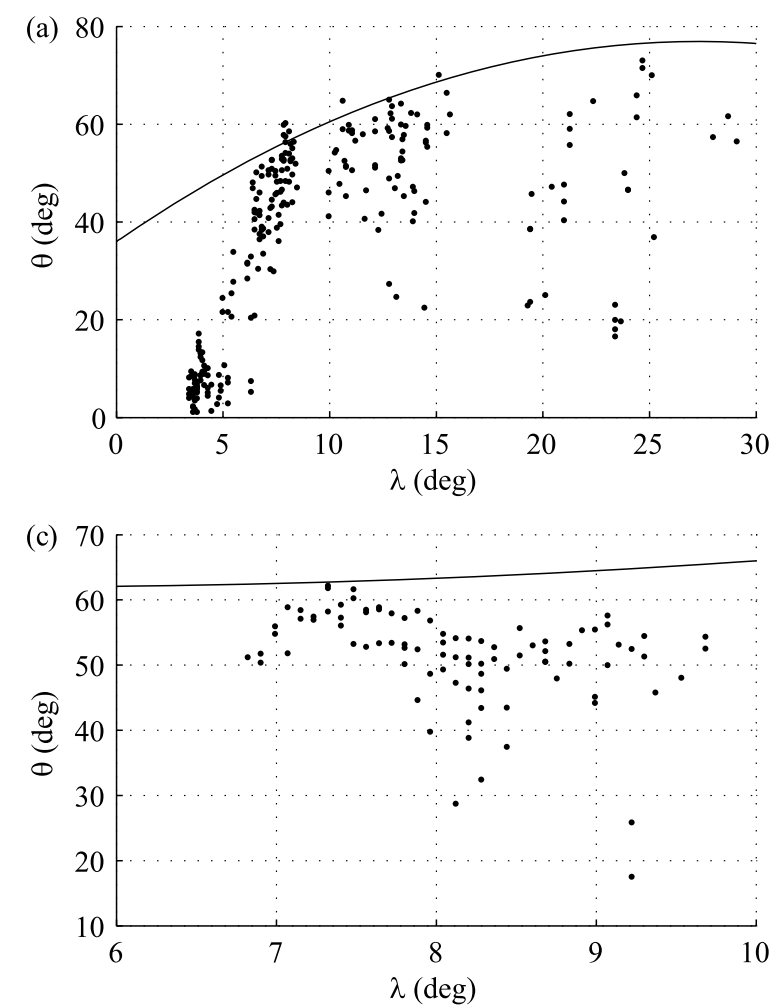

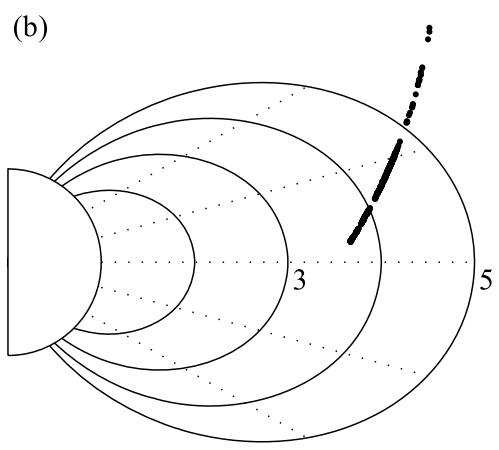

(d)

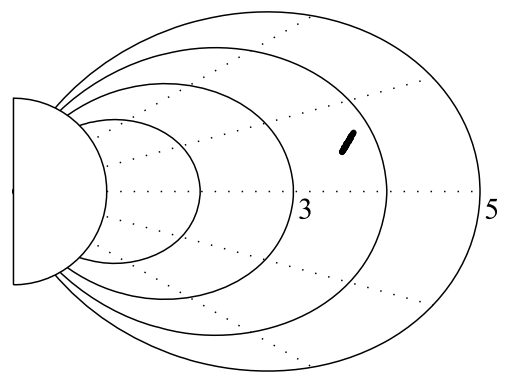

Figure 3. Wave normal angle, $\theta$, versus magnetic latitude, $\lambda$, and the trajectory of the satellite in the meridional plane for two upper band chorus case studies occurring (a and b) on 14 December 1996 from 1906 to 2024 UT near 7.5 MLT and (c and d) on 31 July 1997 from 1601 to 1621 UT near 4.6 MLT. The solid lines in Figures $3 \mathrm{a}$ and $3 \mathrm{c}$ represent the resonance cone angle. The dashed lines in Figures $3 \mathrm{~b}$ and $3 \mathrm{~d}$ represent magnetic latitude increments of $15^{\circ}$, while the solid lines represent dipole $L$ shell increments of $1 R_{\mathrm{E}}$.

included in this study are those for which strong electric fields, corresponding to chorus emissions, were detected. For frequencies below $2 \mathrm{kHz}$, the threshold level is $E>$ $10^{-3} \mathrm{mV}^{2} / \mathrm{m}^{2} / \mathrm{Hz}$. For frequencies above $2 \mathrm{kHz}$, the threshold level is $E>10^{-4.5} \mathrm{mV}^{2} / \mathrm{m}^{2} / \mathrm{Hz}$. After applying the frequency-dependent threshold to all of the orbits, only 10 orbits containing upper band chorus, 12 orbits containing lower band chorus, and 3 orbits containing both upper and lower band chorus remained with sufficient data for wave normal analysis. For each of these orbits, the wave normal angles used for this study will be those that correspond to any frequency that satisfies the frequency-dependent threshold and for which chorus was detected.

[16] The following wave normal analysis will focus exclusively on the polar angle component of the wave normal angle, as the azimuthal angle plays no role in resonant wave-particle interactions, though it is important for determining the propagation of the wave. Though the polar angle can be determined for a range of $0^{\circ}$ to $180^{\circ}$, it will be normalized between $0^{\circ}$ and $90^{\circ}$ such that it is the acute angle with respect to $B_{o}$ in the direction of the Poynting vector.

\section{Upper Band Chorus Wave Normal Angle Analysis}

[17] For the 13 orbits containing upper band chorus waves, two case studies will be shown first, after which statistics for all 13 orbits will be shown. The two case studies can be seen in Figure 3, with the orbit for Figures $3 \mathrm{a}$ and $3 \mathrm{~b}$ occurring on 14 December 1996 and the orbit for Figures 3c and 3d occurring on 31 July 1997 . The case study from 14 December 1996 took place from 1906 to 2024 UT near 7.5 MLT. A portion of the data for this orbit was shown in the sample plot in Figure 2, where it is clear that there are upper band chorus emissions above $0.5 f_{c e-e q}$. Figure $3 \mathrm{a}$ shows the wave normal angles as a function of magnetic latitude. For this case, the wave normal angles are small $(\theta<$ $20^{\circ}$ ) near the equator, but quickly rise to values close to the corresponding resonance cone angle, represented by the solid line, as the satellite moves to higher latitudes. The solid line is the best fit line for the resonance cone angles calculated for each frequency, $\omega$, of the chorus emission that satisfied the frequency-dependent threshold using the formula

$$
\theta_{\text {res }}=\cos ^{-1}\left(\sqrt{\left(\frac{\omega}{\omega_{p}}\right)^{2}+\left(\frac{\omega}{\omega_{c e}}\right)^{2}\left[1-\left(\frac{\omega}{\omega_{p}}\right)^{2}\right]}\right)
$$

where $\omega_{c e}$ and $\omega_{p}$ represent the measured local gyrofrequency and plasma frequency, respectively. Figure $3 \mathrm{~b}$ shows the trajectory of the Polar satellite in the meridional plane for this particular case study. The dashed lines in the meridional plane represent magnetic latitude increments of $15^{\circ}$. The solid lines represent dipole $L$ shell increments of $1 R_{\mathrm{E}}$. The 
(a)

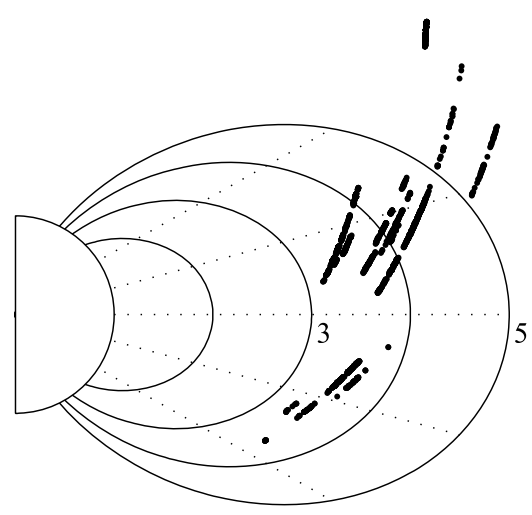

(b)

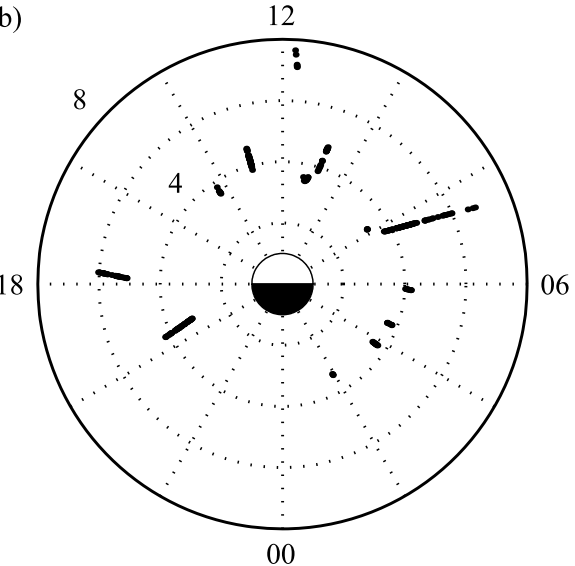

Figure 4. (a) Trajectory of the Polar spacecraft in the meridional plane for the 13 orbits containing upper band chorus emissions. (b) Distribution of MLT and $L$ shells. Each concentric circle represents an increment in $L$ shell by $2 R_{\mathrm{E}}$, while each sector represents an increment in MLT by $2 \mathrm{~h}$.

spacecraft samples magnetic latitudes ranging from $3^{\circ}$ to $29^{\circ}$ and $L$ shells ranging from 3.7 to 6.8 .

[18] The second upper band chorus case study from 31 July 1997 occurred from 1601 to 1621 UT near 4.6 MLT. The latitudinal dependence of the wave normal angles, shown in Figure 3c, illustrate that the wave normal angles remain relatively close to the resonance cone angle for the entire duration of the pass. The satellite samples $6.8^{\circ} \leq \lambda \leq$ $9.8^{\circ}$ and $3.6 \leq L \leq 3.8$.

[19] These two case studies are representative of all of the upper band chorus case studies in this study. The trajectory of the Polar spacecraft for the 13 orbits containing upper band chorus emissions is shown in the meridional plane in Figure $4 \mathrm{a}$. The spacecraft samples a broad range of magnetic latitudes, $3^{\circ} \leq \lambda \leq 35^{\circ}$, and $L$ shells, $3.2 \leq L \leq 7.7$. The distribution of magnetic local times and $L$ shell for the 13 orbits can be seen in Figure 4b. Each concentric circle represents an increment of 2 in $L$ shell, while each sector represents an increment in MLT by $2 \mathrm{~h}$. Because of the relatively sparse sampling in local time, we have not attempted to characterize differences in wave normal angle as a function of that parameter.

[20] The overall distribution of wave normal angles over all magnetic latitudes for all upper band chorus case studies is shown in Figure 5. The histogram is shown for wave normal angles divided into $10^{\circ}$ intervals. The distribution is Gaussian-like, with the peak probability of occurrence taking place between $50^{\circ} \leq \theta<60^{\circ}$. The rise in the probability of occurrence from lower wave normal angles to the peak is much less steep than the fall in the probability of occurrence from the peak to higher wave normal angles.

[21] The probability of occurrence for different ranges of wave normal angles in the three magnetic latitude regions, $\lambda<10^{\circ}, 10^{\circ} \leq \lambda<25^{\circ}$, and $25^{\circ} \leq \lambda<40^{\circ}$, is illustrated in Figure 6. For each magnetic latitude region, the wave normal angles are divided into $10^{\circ}$ intervals with their values indicated by the gray scale coloring. The probability of occurrence for the wave normal angles is normalized with respect to each magnetic latitude region in order to remove the bias of reduced sampling at higher magnetic latitudes by the Polar spacecraft.
[22] As seen in Figure 5, the majority of wave normal angles for upper band chorus occurs between $40^{\circ}$ to $70^{\circ}$ for all latitude ranges. However, Figure 6 demonstrates that there is a slight shift in peak probability of occurrence of the wave normals from higher wave normals $\left(50^{\circ} \leq \theta<60^{\circ}\right)$ at low latitudes $\left(\lambda<10^{\circ}\right)$ to lower wave normals $\left(40^{\circ} \leq \theta<\right.$ $\left.50^{\circ}\right)$ at higher latitudes $\left(25^{\circ} \leq \lambda<40^{\circ}\right)$. This shift in peak can be seen with the help of Figure 7, which shows the difference between the wave normal angle and its corresponding resonance cone angle as a function of magnetic latitude. The resonance cone angle can only be calculated for those orbits for which the electron density can be extracted from the SFR on the Polar spacecraft. Therefore, the magnetic latitude range for Figure 7 is only $4^{\circ} \leq \lambda \leq 27^{\circ}$. In the midlatitude region between $7^{\circ} \leq \lambda \leq 18^{\circ}$, the wave normal angle is very close to the resonance cone angle. However, at higher latitudes, it seems that the wave normal angle starts to move away from the resonance cone angle, though there is reduced sampling by the spacecraft at these latitudes.

[23] In Figure 6, the other significantly high probability of occurrence takes place for wave normal angles in the range $\theta<10^{\circ}$ for magnetic latitudes $\lambda<10^{\circ}$. The case study from 14 December 1996, shown in Figures 3a and 3b,

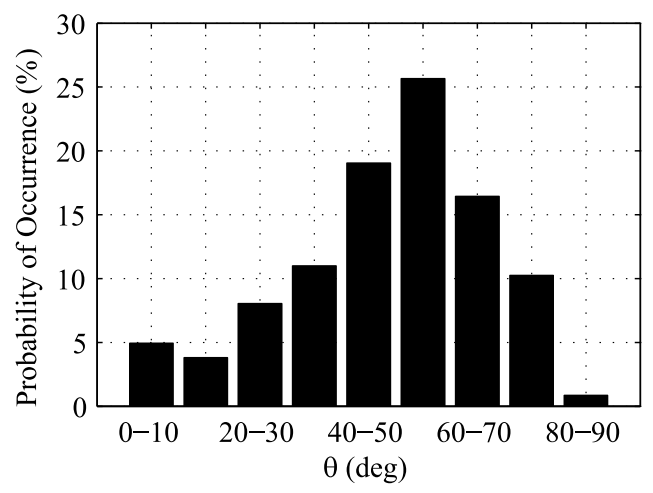

Figure 5. Probability of occurrence for wave normal angle over all magnetic latitudes for upper band chorus case studies. 


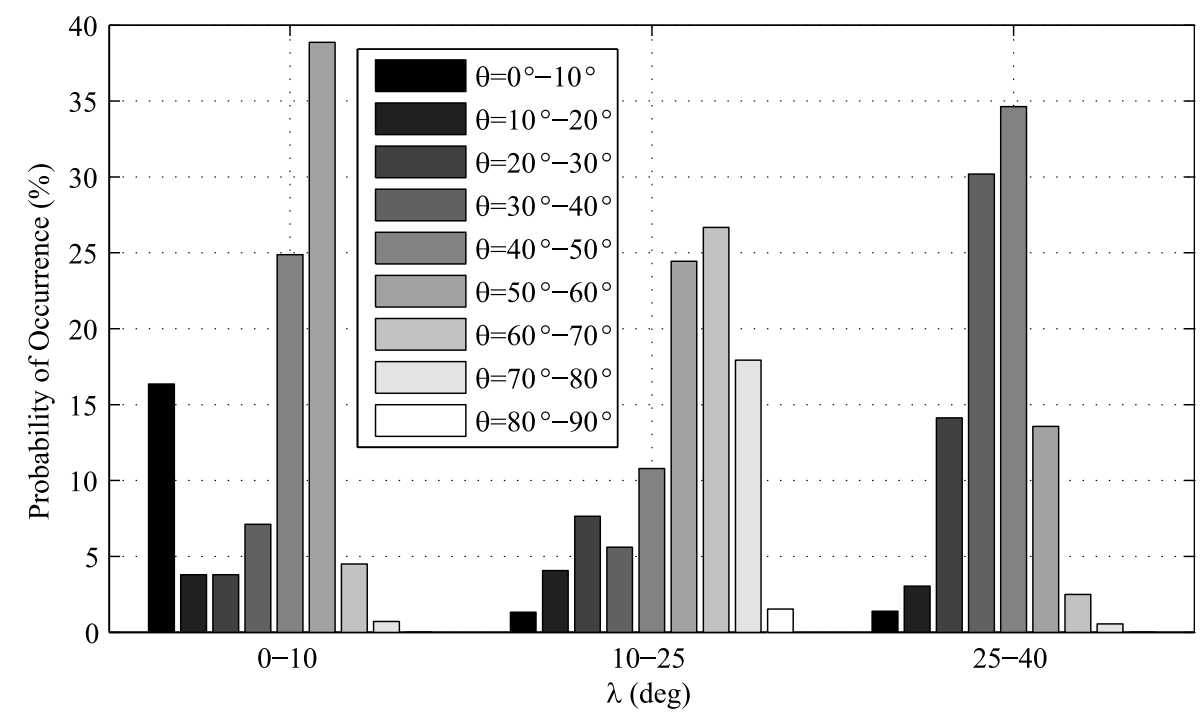

Figure 6. Probability of occurrence for wave normal angles, divided in $10^{\circ}$ intervals, normalized with respect to each magnetic latitude region for upper band chorus emissions. The value of the wave normal angle is indicated by the gray scale coloring.

illustrates this result, as all of the wave normals in this region of high probability are from that orbit and one other orbit on 31 October 1996. The latter pass had very similar characteristics to that of the former, with small wave normal angles near the equator and larger wave normal angles at higher latitudes. This result is consistent with expectations from ray tracing in a smooth magnetosphere. For example, rays launched near the equator outside the plasmapause at a frequency of $0.6 f_{c e-e q}$ and initial wave normal angle parallel to the magnetic field will quickly move toward the resonance cone angle as they propagate to higher magnetic latitudes. As seen in Figure 7, the observations closest to the equator show low wave normals (large difference between $\theta$ and $\theta_{\text {res }}$ ) while observations at latitudes greater than $\sim 7^{\circ}$ show wave normals that are already approaching the resonance cone.

[24] The time-averaged Poynting flux, $S$, was calculated for each upper band case study and is plotted as a function of wave normal angle in Figure 8, with the solid line

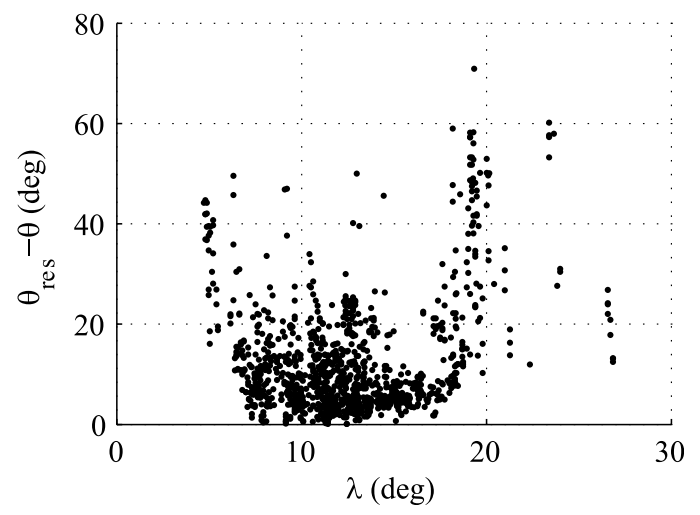

Figure 7. Difference between wave normal angle, $\theta$, and the corresponding resonance cone angle, $\theta_{\text {res }}$, with respect to magnetic latitude for upper band chorus case studies. representing the linear least squares fit to the data. It can be seen that the power ranges from $6.0 \times 10^{-12}$ to $1.7 \times$ $10^{-7} \mathrm{~W} / \mathrm{m}^{2}$ for upper band chorus emissions. Figure 8 also illustrates that the power is almost evenly distributed about its median value $\left(3.1 \times 10^{-10} \mathrm{~W} / \mathrm{m}^{2}\right)$ with no clear dependence on the wave normal angle.

\section{Lower Band Chorus Wave Normal Angle Analysis}

[25] For the 15 orbits containing lower band chorus waves, two case studies will be shown first, after which overall statistics will be shown. The two case studies can be seen in Figure 9, with the orbit for Figures 9a and 9b occurring on 28 February 1997 and the orbit for Figures $9 c$ and $9 \mathrm{~d}$ occurring on 14 September 1997 . The case study from 28 February 1997 took place from 1015 to 1041 UT near 2.8 MLT. The latitudinal dependence of the wave normal angle in Figure 9a shows the vast amount of scatter in the

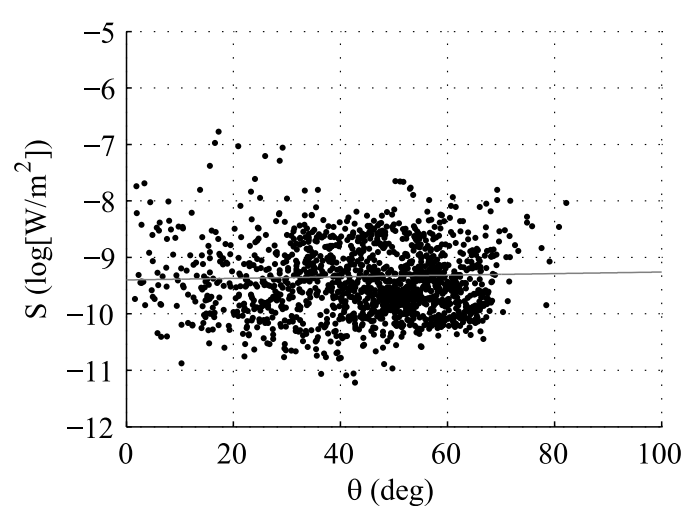

Figure 8. Time-averaged Poynting flux, $S$, as a function of wave normal angle for upper band chorus emissions. The solid line represents the linear least squares fit to the data. 

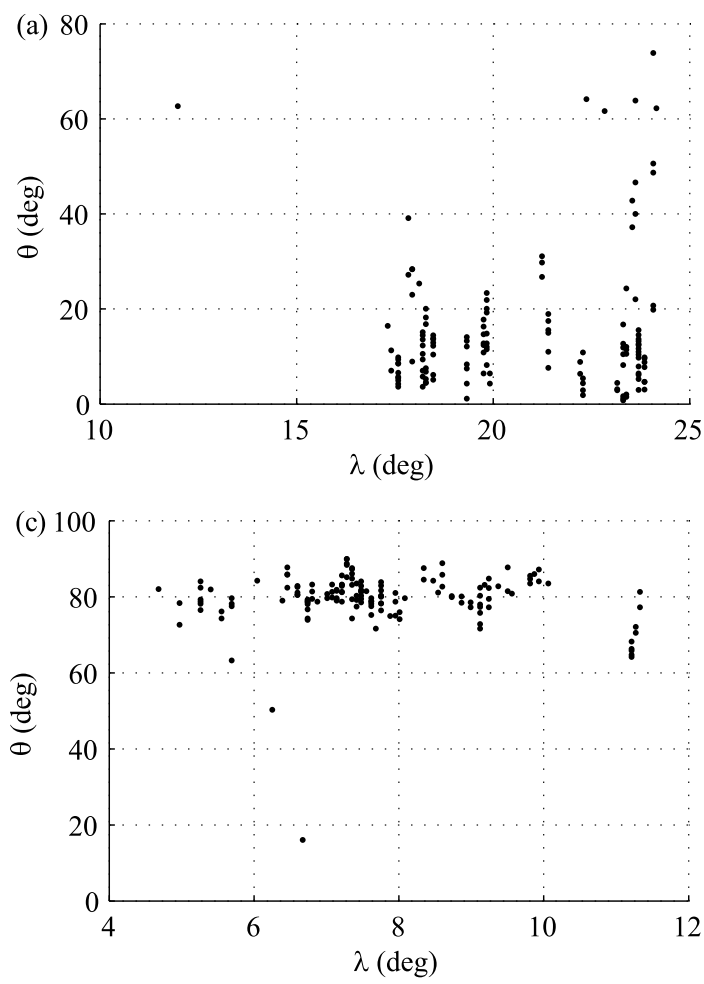

(b)

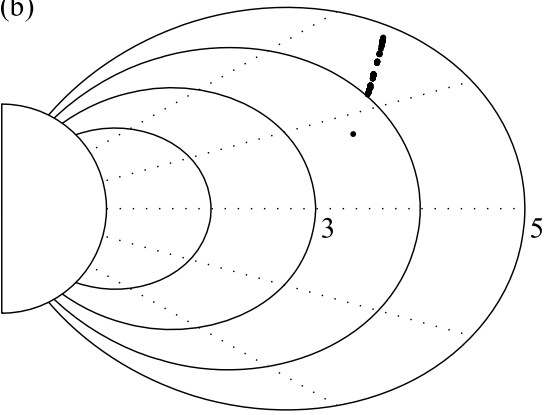

(d)

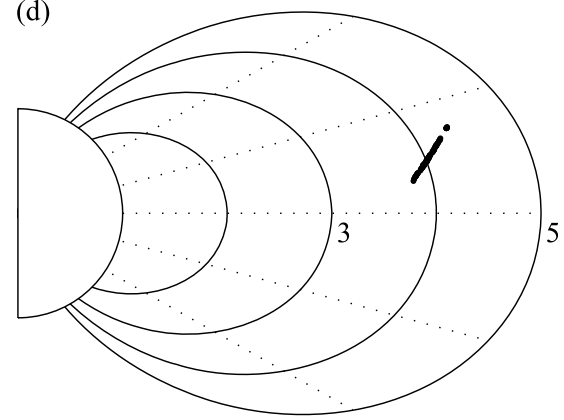

Figure 9. Wave normal angle versus magnetic latitude and the trajectory of the satellite in the meridional plane for two lower band chorus case studies occurring ( $a$ and b) on 28 February 1997 from 1015 to 1041 UT near 2.8 MLT and (c and d) on 14 September 1997 from 1510 to 1530 UT near 1.8 MLT.

wave normal angles with respect to magnetic latitude typical of nearly all the orbits containing lower band chorus. The Polar spacecraft samples magnetic latitudes ranging from $10^{\circ}$ to $25^{\circ}$ and $L$ shells ranging from 3.5 to 5.0 , as seen in its trajectory in the meridional plane in Figure $9 \mathrm{~b}$.

[26] The second lower band chorus case study from 14 September 1997 occurred from 1510 to 1530 UT near 1.8 MLT. This case study is an exception from all of the other Polar orbits containing lower band chorus. First, there is an absence of scatter in the latitudinal dependence of the wave normal angles, shown in Figure 9c, as compared with the other 14 lower band case studies. Second, nearly all of the wave normal angles have values of $70^{\circ}<\theta \leq 90^{\circ}$ for the magnetic latitudes sampled. The trajectory of the Polar spacecraft, which samples $4^{\circ} \leq \lambda \leq 12^{\circ}$ and $3.8 \leq L \leq 4.4$, in the meridional plane is shown in Figure 9d. Unfortunately, this is also the only lower band case study within $10^{\circ}$ of the equatorial plane. This unusual case study is shown for completeness, but will be excluded from the overall statistics. Consequently, the statistics shown in Figures 9 through 14 will consist of data only from the remaining 14 orbits. (a)

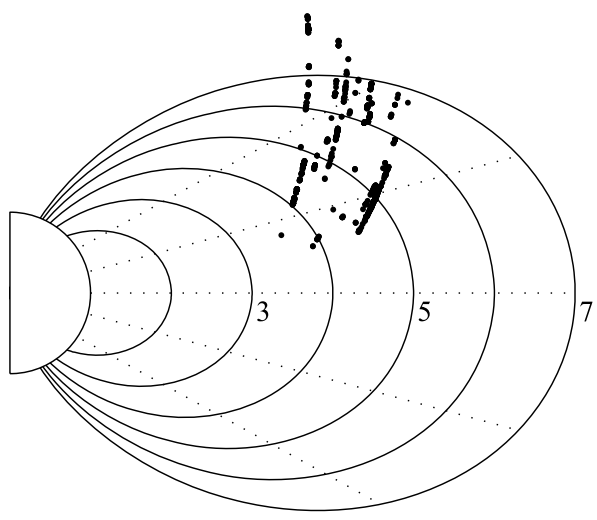

(b)

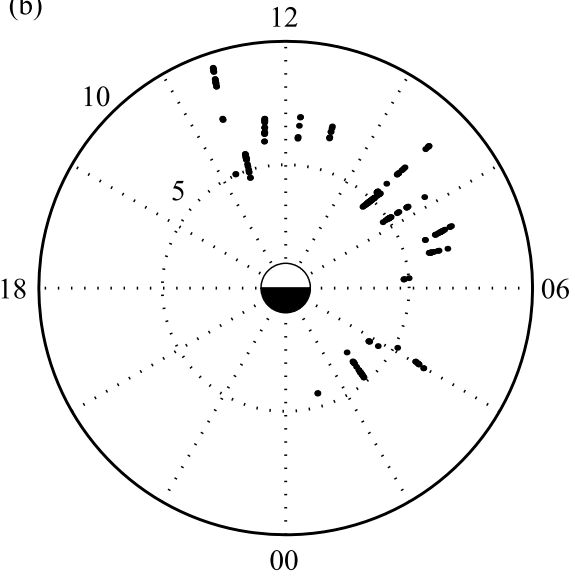

Figure 10. (a) Trajectory of the Polar spacecraft in the meridional plane for the 14 orbits containing lower band chorus emissions. (b) Distribution of MLT and $L$ shells. 


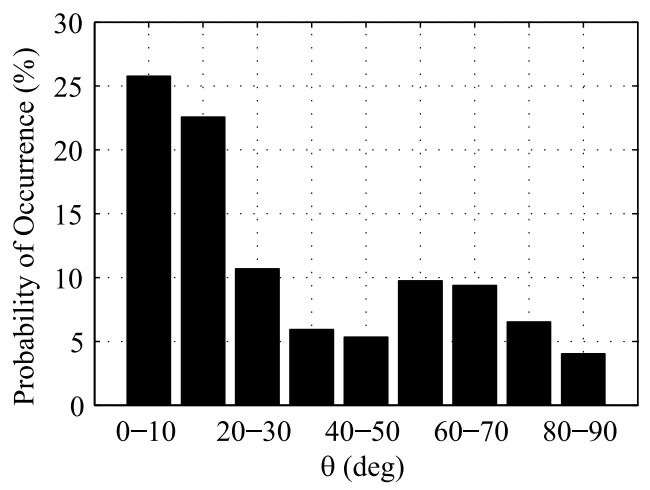

Figure 11. Probability of occurrence for wave normal angle over all magnetic latitudes for lower band chorus case studies.

[27] The trajectory of the Polar spacecraft for the remaining 14 orbits containing lower band chorus emissions is shown in the meridional plane in Figure 10a. The spacecraft samples a broad range of magnetic latitudes, $8^{\circ} \leq \lambda \leq 43^{\circ}$, and $L$ shells, $3.6 \leq L \leq 9.4$. The distribution of magnetic local times, ranging from 1.1 to 13.6 , and $L$ shells for the 14 orbits can be seen in Figure 10b.

[28] The overall distribution of wave normal angles over all magnetic latitudes for the 14 lower band case studies is illustrated in Figure 11. The distribution is uneven, with the majority of wave normal angles having values less than $20^{\circ}$ and a secondary peak with lower overall probability in the range of $50^{\circ}-60^{\circ}$.

[29] Figure 12 illustrates the probability of occurrence for different ranges of wave normal angles with respect to three magnetic latitude regions, $10^{\circ} \leq \lambda<25^{\circ}, 25^{\circ} \leq \lambda<40^{\circ}$, and $40^{\circ} \leq \lambda<50^{\circ}$. As mentioned earlier, the case study from 14 September 1997 is not included in the overall statistical analysis, and thus there is not sufficient data left in the magnetic latitude region $\lambda<10^{\circ}$. For each of the other magnetic latitude regions, the probability of occurrence for

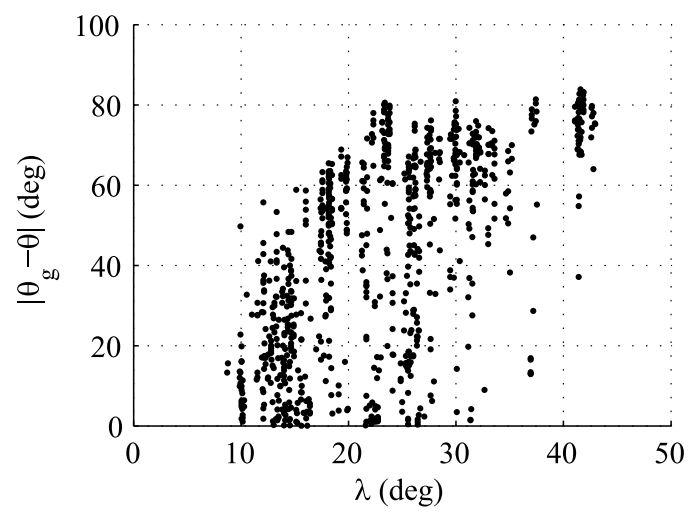

Figure 13. Absolute value of the difference between wave normal angle, $\theta$, and the corresponding Gendrin angle, $\theta_{g}$, with respect to magnetic latitude for lower band chorus case studies.

the wave normal angles is normalized with respect to each latitude region.

[30] It can be seen in Figure 12 that the probability of occurrence of wave normal angles in the range $\theta<20^{\circ}$ increases with increasing latitude, $\lambda$. Contrarily, the higher wave normal angles observed near the equator have a decreasing relative probability with increasing latitude.

[31] Figure 13 shows the absolute value of the difference between the wave normal angle and its corresponding Gendrin angle, defined as $\theta_{g}=\cos ^{-1}\left(\frac{2 \omega}{\omega_{c e}}\right)$, as a function of magnetic latitude. For latitudes less than $25^{\circ}, 20 \%$ of the wave normal angles are within $10^{\circ}$ of $\theta_{g}$. These wave normal angles move further away from the Gendrin angle with increasing latitude.

[32] Figure 14 illustrates the dependence of the timeaveraged Poynting flux, $S$, on wave normal angle. It can be seen from Figure 14 that lower band chorus emissions have power levels ranging from $6.9 \times 10^{-10}$ to $1.5 \times 10^{-6} \mathrm{~W} / \mathrm{m}^{2}$. As the value of the wave normal angle increases, the

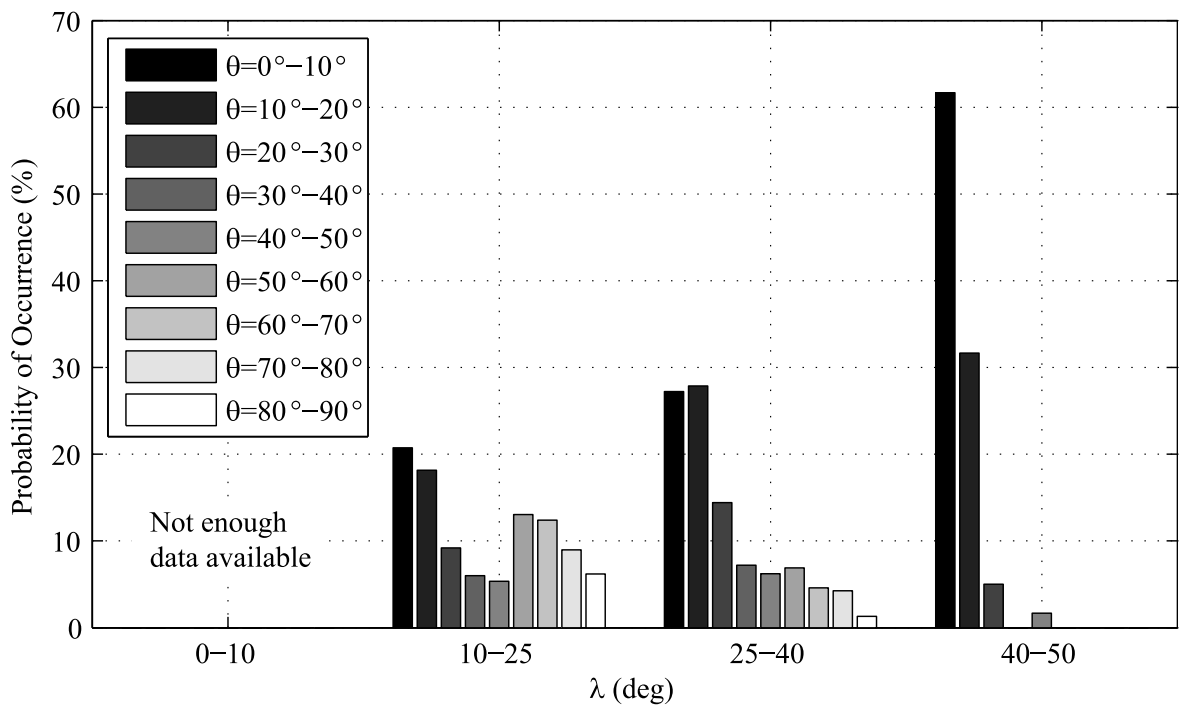

Figure 12. Probability of occurrence for wave normal angles, divided in $10^{\circ}$ intervals, normalized with respect to each magnetic latitude region for lower band chorus emissions. 


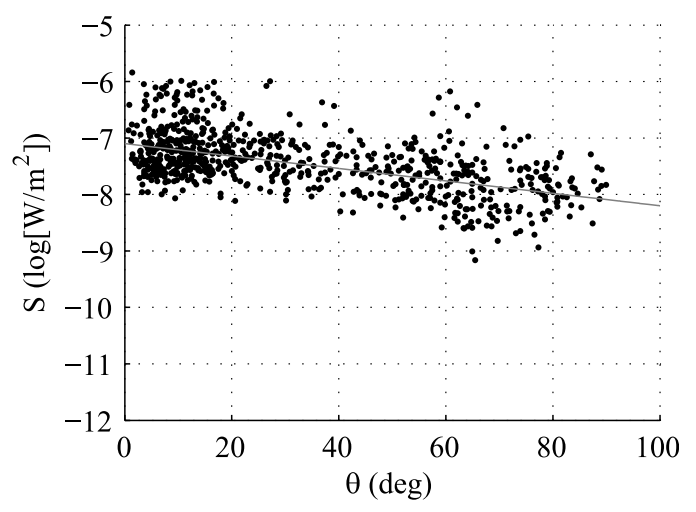

Figure 14. Time-averaged Poynting flux, $S$, as a function of wave normal angle for lower band chorus emissions. The solid line represents the linear least squares fit to the data.

wave power decreases, which can be seen by the solid line representing the linear least squares fit to the data.

\section{Discussion}

[33] The results from the upper and lower band chorus case studies can be compared to the previous work done on these types of emissions. For the upper band chorus case studies, $50 \%$ of the wave normal angles at latitudes near the magnetic equator $\left(\lambda \leq 7^{\circ}\right)$ have values less than $10^{\circ}$. These results for low latitudes are consistent with those of Hospodarsky et al. [2001], which stated that the wave propagates primarily parallel to the magnetic field for $\lambda<$ $5^{\circ}$. They also agree with the conclusion of Lauben et al. [2002] that the wave normal angles in the equatorial source region have values $\theta \simeq 0^{\circ}$. For some of the other case studies, the wave normal angles are close in value to their corresponding resonance cone angles for all of the magnetic latitudes sampled. This finding agrees with that of Hayakawa et al. [1984], which shows $\theta \sim \theta_{\text {res }}$ near the magnetic equator.

[34] The wave normal angle is very close to the resonance cone angle for all of the upper band chorus emissions in the midlatitude region between $7^{\circ} \leq \lambda \leq 18^{\circ}$. This finding is in agreement with the finding of Muto et al. [1987] that $\theta \simeq$ $\theta_{\text {res }}$ at $\lambda \sim 17^{\circ}$ and the earlier stated results of Hayakawa et al. [1984]. Overall, our results are consistent with the assertion of Lauben et al. [2002] that upper band chorus is emitted near the equator at $\theta \simeq 0^{\circ}$, but that propagation away from the equator quickly moves the wave normal angles close to the resonance cone.

[35] At higher latitudes, $\lambda>18^{\circ}$, the wave normal angles of upper band chorus are observed to move away from the resonance cone by an average value of $30^{\circ}$. This effect was also reported by Muto et al. [1987]. It is likely that the lack of high wave normals at higher latitudes is the result of Landau damping [Lauben et al., 2002]. Waves near the resonance cone are expected to be strongly Landau damped as they propagate [Burton and Holzer, 1974]. Thus, the only waves with observable amplitudes at the higher latitudes are those with wave normals away from the resonance cone.
[36] For the unusual lower band case study, occurring on 14 September 1997, it can be seen that the wave normal angles are grouped around $\theta=80^{\circ}$ for magnetic latitudes less than approximately $12^{\circ}$. This case is in stark contrast to previous reports on lower band chorus [e.g., Burton and Holzer, 1974; Goldstein and Tsurutani, 1984; Hayakawa et al., 1984; Hospodarsky et al., 2001], and as far as these authors are aware, it is the only reported case of lower band chorus of this type.

[37] At all latitudes sampled by Polar, lower band chorus has the highest probability of occurrence for $\theta<20^{\circ}$. In addition, as the waves propagate to higher magnetic latitudes, the relative probability of observing chorus for $\theta<$ $20^{\circ}$ increases. For example, for $10^{\circ} \leq \lambda<25^{\circ}$, wave normals have values less than $20^{\circ}$ for $\sim 40 \%$ of the time, while for $\lambda$ $\geq 40^{\circ}$ the occurrence is greater than $90 \%$. This result might be explained by the suggestion by Burton and Holzer [1974] that ducts are necessary to allow lower band waves to reach latitudes in the range of $40^{\circ}-50^{\circ}$. The small values of wave normal angles at higher magnetic latitudes may also be explained by the unducted propagation of waves with high wave normal angles, near the Gendrin angle, directed toward the Earth in the source region [Chum and Santolik, 2005; Santolik et al., 2006].

[38] In contrast, wave normal angles with values $\theta \geq 20^{\circ}$ have a decreasing probability of occurrence as the lower band chorus wave propagates to higher magnetic latitudes. According to Lauben et al. [2002], waves observed on the Polar spacecraft with high wave normal angles will soon be Landau damped at higher magnetic latitudes. In support of this, Burton and Holzer [1974] state that wave normal angles with values greater than $60^{\circ}$ will rarely be detected at high latitudes due to strong attenuation. Thus, before the Polar spacecraft is able to sample the high magnetic latitudes, the waves will have been strongly damped, explaining the absence of high wave normal angles in this region.

[39] For the lower band chorus emissions, it was seen that the portion of wave normal angles that start near the Gendrin angle tend to move away from the Gendrin angle with increasing values of magnetic latitude. It is possible that some fraction of lower band chorus waves are generated at the Gendrin angle within the source region as was concluded by Lauben et al. [2002].

[40] For each of the orbits of both types of emissions, the wave normal angles used for this study were those that corresponded to any frequency that satisfied the frequencydependent threshold and for which chorus was detected. There does exist a small variation in wave normal angle with respect to frequency, though there is no systematic trend to this variation. Specifically, at any give time step, the average spread of the wave normal angle over frequency is $\sim 2^{\circ}$ for upper band chorus and $\sim 8^{\circ}$ for lower band chorus.

[41] The time-averaged Poynting flux was calculated for both upper and lower band chorus emissions, illustrating that the upper band chorus waves have power levels that are 60 times smaller than those of the lower band chorus waves on average. This difference is supported by past work, which states that the lower band of chorus is more intense than the upper band [Bortnik et al., 2008]. The trend for each band is also different, with the power distributed about its median value for upper band chorus waves and an 
inversely proportional relationship between the power and wave normal angle for lower band chorus waves.

\section{Conclusion}

[42] Data from the High Frequency Waveform Receiver on board the Polar spacecraft was used to analyze 1,765 and 993 wave normal angles from 13 orbits containing upper band magnetospheric chorus emissions and 15 orbits containing lower band magnetospheric chorus emissions, respectively. Due to the highly elliptical orbit of the Polar spacecraft, it is impossible to distinguish between latitudinal and $L$ shell variations in the distribution of wave normal angles. However, since the effect of the propagation of waves from the equatorial source region to the off-equatorial region is expected to be much stronger on the wave normal distribution than variations with $L$ shell, the distribution of wave normal angles in this study were characterized as a function of magnetic latitude only.

[43] For upper band chorus emissions, two types of wave normal angles were seen: (1) wave normal angles with values $\theta<20^{\circ}$ for magnetic latitudes $\lambda<10^{\circ}$, which then quickly rise to values close to the resonance cone with increasing latitude, and (2) wave normal angles that remain near the resonance cone angle for $7^{\circ} \leq \lambda \leq 18^{\circ}$. For either type, the wave normal angle moves away from the resonance cone angle at high latitudes, $\lambda>18^{\circ}$, by an average of $30^{\circ}$.

[44] For lower band chorus emissions, the following conclusions about the wave normal angles can be made: (1) wave normal angles tend to be scattered with respect to magnetic latitude and the majority of the wave normal angles occur in the range $\theta<20^{\circ},(2)$ wave normal angles have values $\theta<20^{\circ}$ with increasing probabilities of occurrence as magnetic latitude increases, (3) wave normal angles have values $\theta \geq 20^{\circ}$ with decreasing probabilities of occurrence as magnetic latitude increases, and (4) for magnetic latitudes less than $25^{\circ}$, $20 \%$ of the wave normal angles are within $10^{\circ}$ of the Gendrin angle.

[45] Regarding the time-averaged Poynting flux, the following trends were found: (1) lower band chorus waves have power levels that are 60 times larger on average than upper band chorus waves, (2) the time-averaged Poynting flux is fairly evenly distributed about its median value for all values of wave normal angle for upper band chorus emissions, and (3) the time-averaged Poynting flux decreases with respect to wave normal angle for lower band chorus emissions.

[46] The conclusions from this study establish a broader understanding of the distribution of chorus wave normal angles. These results will be important for further studies of resonant interactions between chorus waves and electrons over a wide range of energies.

[47] Acknowledgments. The work at Stanford University was supported by the Office of Naval Research under grant Z882802-A and by NASA under grants NNX07AI24G and NNX09AF51G. The work at the University of Iowa was supported by the NASA LWS program under JPL subcontract 1346597 and by NASA grant NNG05GM52G. O.S. acknowledges support from NSF award 0307319/KONTAKT ME842 and GAAV grant IAA301120601.

[48] Zuyin Pu thanks Michael Starks and George Hospodarsky for their assistance in evaluating this paper.

\section{References}

Allcock, G. M. (1957), A study of the audio-frequency radio phenomenon known as 'dawn chorus,' Aust. J. Phys., 10, 286-298.

Bortnik, J., R. M. Thorne, and N. P. Meredith (2008), The unexpected origin of plasmaspheric hiss from discrete chorus emissions, Nature, 452(7183), 62, doi:10.1038/nature06741.

Burtis, W. J., and R. A. Helliwell (1969), Banded chorus: A new type of VLF radiation observed in the magnetosphere by OGO 1 and OGO 3, J. Geophys. Res., 74, 3002-3010.

Burtis, W. J., and R. A. Helliwell (1976), Magnetospheric chorus: Occurrence patterns and normalized frequency, J. Geophys. Res., 24, 10071024.

Burton, R. K., and R. E. Holzer (1974), The origin and propagation of chorus in the outer magnetosphere, J. Geophys. Res., 79, 1014-1023.

Chum, J., and O. Santolík (2005), Propagation of whistler-mode chorus to low altitudes: Divergent ray trajectories and ground accessibility, Ann. Geophys., 23, 3727-3738.

Dunckel, N., and R. A. Helliwell (1969), Whistler-mode emissions on the OGO 1 satellite, J. Geophys. Res., 74(26), 6371-6385.

Goldstein, B. E., and B. T. Tsurutani (1984), Wave normal directions of chorus near the equatorial source region, J. Geophys. Res., 89, 2789 2810.

Gurnett, D. A., and B. J. O’Brien (1964), High-latitude geophysical studies with satellite Injun 3: 5. Very low frequency electromagnetic radiation, J. Geophys. Res., 69, 65-89.

Gurnett, D. A., et al. (1995), The Polar plasma wave instrument, Space Sci. Rev., 71, 597-622.

Hayakawa, M., Y. Yamanaka, M. Parrot, and F. Lefeuvre (1984), The wave normals of magnetospheric chorus emissions observed on board GEOS 2, J. Geophys. Res., 89, 2811-2821.

Helliwell, R. A. (1965), Whistlers and Related Ionospheric Phenomena, Stanford Univ. Press, Stanford, Calif.

Helliwell, R. A. (1969), Low-frequency waves in the magnetosphere, Rev. Geophys., 71(1), 281-303.

Horne, R. B., and R. M. Thorne (1998), Potential waves for relativistic electron scattering and stochastic acceleration during magnetic storms, Geophys. Res. Lett., 25(15), 3011-3014.

Horne, R. B., R. M. Thorne, S. A. Glauert, J. M. Albert, N. P. Meredith, and R. R. Anderson (2005a), Timescale for radiation belt electron acceleration by whistler mode chorus waves, J. Geophys. Res., 110, A03225, doi:10.1029/2004JA010811.

Horne, R. B., et al. (2005b), Wave acceleration of electrons in the Van Allen radiation belts, Nature, 437(7056), 227-230, doi:10.1038/nature03939.

Hospodarsky, G. B., T. F. Averkamp, W. S. Kurth, D. A. Gurnett, M. Dougherty, U. Inan, and T. Wood (2001), Wave normal and Poynting vector calculations using the Cassini radio and plasma wave instrument, J. Geophys. Res., 106(A12), 30,253-30,269.

Inan, U. S., Y. T. Chiu, and G. T. Davidson (1992), Whistler-mode chorus and morningside aurorae, Geophys. Res. Lett., 19, 653-656.

Kennel, C. F., and H. E. Petschek (1966), Limit on stably trapped particle fluxes, J. Geophys. Res., 71, 1-28.

Lauben, D. S., U. S. Inan, T. F. Bell, and D. A. Gurnett (2002), Source characteristics of ELF/VLF chorus, J. Geophys. Res., 107(A12), 1429, doi:10.1029/2000JA003019.

LeDocq, M. J., D. A. Gurnett, and G. B. Hospodarsky (1998), Chorus source locations from VLF Poynting flux measurements with the POLAR spacecraft, Geophys. Res. Lett., 25, 4063-4066.

Lorentzen, K. R., J. B. Blake, U. S. Inan, and J. Bortnik (2001), Observations of relativistic electron microbursts in association with VLF chorus, J. Geophys. Res., 106(A4), 6017-6027.

Mosier, S. R., M. L. Kaiser, and L. W. Brown (1973), Observations of noise bands associated with the upper hybrid resonance by the Imp 6 radio astronomy experiment, J. Geophys. Res., 78(10), 1673-1679.

Muto, H., M. Hayakawa, M. Parrot, and F. Lefeuvre (1987), Direction finding of half-gyrofrequency VLF emissions in the off-equatorial region of the magnetosphere and their generation and propagation, J. Geophys. Res., 92, 7538-7550.

Ni, B., R. M. Thorne, Y. Y. Shprits, and J. Bortnik (2008), Resonant scattering of plasma sheet electrons by whistler-mode chorus: Contribution to diffuse auroral precipitation, Geophys. Res. Lett., 35, L11106, doi:10.1029/2008GL034032.

Parrot, M., O. Santolík, N. Cornilleau-Wehrlin, M. Maksimovic, and C. C. Harvey (2003), Source location of chorus emissions observed by Cluster, Ann. Geophys., 21, 473-480.

Rosenberg, T. J., R. A. Helliwell, and J. P. Katsufrakis (1971), Electron precipitation associated with discrete very-low-frequency emissions, J. Geophys. Res., 76(34), 8445-8452. 
Rosenberg, T. J., J. C. Siren, D. L. Matthews, K. Marthinsen, J. A. Holtet, A. Egeland, D. L. Carpenter, and R. A. Helliwell (1981), Conjugacy of electron microbursts and VLF chorus, J. Geophys. Res., 86(A7), 5819-5832.

Rosenberg, T. J., R. Wei, D. L. Detrick, and U. S. Inan (1990), Observations and modeling of wave-induced microburst electron precipitation, J. Geophys. Res., 95, 6467-6475.

Russell, C. T., R. C. Snare, J. D. Means, D. Pierce, D. Dearborn, M. Larson, G. Barr, and G. Le (1995), The GGS/Polar magnetic fields investigations, Space Sci. Rev., 71, 563-582.

Santolík, O., and D. A. Gurnett (2002), Propagation of auroral hiss at high altitudes, Geophys. Res. Lett., 29(10), 1481, doi:10.1029/ 2001 GL013666.

Santolík, O., J. S. Pickett, D. A. Gurnett, and L. R. O. Storey (2002), Magnetic component of narrowband ion cyclotron waves in the auroral zone, J. Geophys. Res., 107(A12), 1444, doi:10.1029/2001JA000146.

Santolík, O., M. Parrot, and F. Lefeuvre (2003), Singular value decomposition methods for wave propagation analysis, Radio Sci., 38(1), 1010 doi:10.1029/2000RS002523.

Santolík, O., D. A. Gurnett, J. S. Pickett, M. Parrot, and N. CornilleauWehrlin (2005), Central position of the source region of storm-time chorus, Planet. Space Sci., 53, 299-305, doi:10.1016/j.pss.2004.09.056.

Santolík, O., J. Chum, M. Parrot, D. A. Gurnett, J. S. Pickett, and N. Cornilleau-Wehrlin (2006), Propagation of whistler mode chorus to low altitudes: Spacecraft observations of structured ELF hiss, J. Geophys. Res., 111, A10208, doi:10.1029/2005JA011462.

Sazhin, S. S., and M. Hayakawa (1992), Magnetospheric chorus emissions: A review, Planet. Space Sci., 40, 681-697.

Shprits, Y. Y., R. M. Thorne, R. B. Horne, S. A. Glauert, M. Cartwright C. T. Russell, D. N. Baker, and S. G. Kanekal (2006), Acceleration mechanism responsible for the formation of the new radiation belt during the 2003 Halloween solar storm, Geophys. Res. Lett., 33, L05104, doi:10.1029/2005GL024256.

Storey, L. R. O. (1953), An investigation of whistling atmospherics, Philos. Trans. R. Soc. London, Ser. A, 246, 113-141.

Summers, D., B. Ni, and N. P. Meredith (2007a), Timescales for radiation belt electron acceleration and loss due to resonant wave-particle interactions: 1. Theory, J. Geophys. Res., 112, A04206, doi:10.1029/2006JA011801.

Summers, D., B. Ni, and N. P. Meredith (2007b), Timescales for radiation belt electron acceleration and loss due to resonant wave-particle interactions: 1. Evaluation for VLF chorus, ELF hiss, and electromagnetic ion cyclotron waves, J. Geophys. Res., 112, A04207, doi:10.1029/ 2006JA011993.

Thorne, R. M., T. P. O’Brien, Y. Y. Shprits, D. Summers, and R. B. Horne (2005), Timescale for MeV electron microburst loss during geomagnetic storms, J. Geophys. Res., 110, A09202, doi:10.1029/2004JA010882.

Tsurutani, B. E., and E. J. Smith (1974), Postmidnight chorus: A substorm phenomenon, J. Geophys. Res., 79, 118-127.

Villalon, E., and W. J. Burke (1995), Pitch angle scattering of diffuse auroral electrons by whistler mode waves, J. Geophys. Res., 100(A10), $19,361-19,370$

N. Haque, U. S. Inan, and M. Spasojevic, Space, Telecommunications, and Radioscience Laboratory, Stanford University, 350 Serra Mall, Stanford, CA 94305, USA. (naoshin@stanford.edu; inan@stanford.edu; mariaspasojevic@stanford.edu)

O. Santolík, Department of Space Physics, Institute of Atmospheric Physics, Bocni II/1401, 14131 Prague 4, Czech Republic. (ondrej.santolik@ mff.cuni.cz) 